\title{
Simulation des essais cliniques dans le développement des médicaments
}

\author{
Pascal Girard, ${ }^{1}$ Michel Cucherat, ${ }^{2}$ David Guez ${ }^{3}$ et les participants à la Table Ronde $n^{\circ} 2$ \\ de Giens XIX ${ }^{\dagger}$
}

1 INSERM et EA 3738 Ciblage Thérapeutique en Oncologie, Faculté de Médecine Lyon-Sud, Lyon, France

2 Hôpital Laennec, Pharmacologie EA 643, Lyon, France

3 Laboratoires Servier, Neuilly-sur-Seine, France

\section{Résumé}

\begin{abstract}
Simulation du modèle thérapeutique et simulation d'essais cliniques ont récemment retenu l'attention comme techniques émergentes pour l'aide au développement de nouvelles molécules et l'exploration des résultats d'essais cliniques. Ces approches bénéficient des progrès récents dans le domaine des modèles « in silico » ainsi que des progrès dans celui des modèles non linéaires à effets mixtes appliqués aux modèles pharmacocinétiquespharmacodynamiques. La fusion des deux approches permet la simulation de patients « virtuels » qui recevront un traitement ou un placebo. Ces techniques peuvent être utilisées comme «preuve du concept», pour l'analyse de la décision ou l'optimisation de plans expérimentaux. Egalement, l'impact des écarts au protocole sur les résultats d'un essai clinique peut facilement être évalué par simulation. Cette technique commence à être implémentée par l'industrie pharmaceutique pour l'optimisation des plans expérimentaux en phases II et III quand un modèle pour un «biomarqueur » ou un effet clinique existe; par contre l'utilisation du modèle thérapeutique « in silico » comme « preuve du concept» n'en est qu'à ses débuts. Afin de diffuser ces méthodologies plus largement dans le cadre du développement du médicament, des travaux multidisciplinaires sont nécessaires, de nouveaux outils de modélisation et de simulation doivent être développés et des documents de bonnes pratiques de modélisation et simulation adoptés. La réduction du nombre d'échecs lors du développement d'un nouveau médicament, du nombre d'études négatives en phases II et III des essais cliniques, ou simplement la réduction de leurs durées et de leurs coûts sont parmi les bénéfices attendus d'une extension de la modélisation et de la simulation en pharmacologie clinique.
\end{abstract}

\section{Introduction}

Le sujet de la table ronde ${ }^{\circ} 2$ de Giens 2004 était la simulation d'essais cliniques, technique émergente qui a récemment retenu l'attention pour faciliter la synthèse des connaissances et l'exploration des résultats possibles d'essais cliniques sur la base de modèles mathématiques/stochastiques de l'action du médicament et de la progression de la maladie combinés avec un modèle décrivant l'essai clinique. ${ }^{[1-7]}$ Cette approche bénéficie des progrès fondamentaux dans le développement des modèles « in silico » comme le projet Physiome, ${ }^{[8]}$ ainsi que des progrès dans le domaine des modèles non linéaires à effets mixtes qui, s'ils sont plus éloignés des processus physiologiques, permettent la prise en compte de différentes sources de variabilités (inter- et intra-patients) et d'incertitudes. ${ }^{[9]}$ Le mélange de ces deux approches permet la simulation de patients « virtuels » qui peuvent être utilisés pour divers objectifs comme établir la « preuve de concept », pour une démarche «d'analyse de la décision » ou l'optimisation d'un plan expérimental. Le terme « virtuel » est ici mis entre guillemets car, ainsi que ce fut discuté au cours de la table ronde, le «patient virtuel » relève encore d'un concept de science fiction. Il faut toutefois remarquer que si cet outil n'en est qu'à ses débuts en pharmacologie clinique, il est largement utilisé dans d'autres domaines industriels comme la conception automobile ou aéronautique et les simulateurs de vol pour la conception des avions et l'entraînement des pilotes. ${ }^{[10]}$ La table

$\dagger$ Pour la liste des participants, voir en fin d'article. 
ronde a exploré ces différents concepts et définitions de la simulation des essais cliniques, ces avantages et limitations ainsi que son domaine d'application et son actuelle pénétration dans le domaine de l'industrie pharmaceutique comme dans celui universitaire en France.

\section{Définitions}

\subsection{Définitions générales}

Avant toute considération sur la simulation d'essais cliniques, la table ronde s'est attachée à la définition/clarification de certains concepts.

- Modèle : représentation mathématique du comportement d'un système.

- Simulation : étude du comportement d'un système à l'aide d'une implémentation informatique d'un modèle mathématique.

- Simulation d'essais cliniques : étude de certains résultats d'un essai clinique, à l'aide de l'implémentation informatique d'un modèle mathématique et du plan expérimental de l'étude.

Les modèles sont essentiellement de deux types, descriptif ou mécanistique, avec un certain continuum entre les deux. ${ }^{[11,12]}$ L'utilisation de modèles mécanistiques permet la définition d'un second type de simulation, la simulation du modèle thérapeutique. Les caractéristiques essentielles de ces deux types de modèles et concepts de simulation sont maintenant décrites plus en détail.

\subsection{Modèles descriptifs}

Les modèles descriptifs sont une représentation mathématique du comportement d'un système dont l'intérêt est d'ajuster les données et d'en proposer un résumé à travers un jeu réduit de paramètres. Ces modèles ignorent totalement les mécanismes et la physiologie sous-jacente des phénomènes biologiques qu'ils décrivent. Des exemples types sont les modèles polynomiaux ou les «spline » qui peuvent s'ajuster à n'importe quel type de courbe non linéaire. ${ }^{[13]}$ Leurs principaux avantages sont la flexibilité, le fait qu'ils soient des modèles génériques, leur simplicité et facilité d'implémentation. Les principales limites sont tout d'abord les mauvaises propriétés d'interpolation/extrapolation, puisqu'en dehors des concentrations et/ou doses observées, ces modèles ont des capacités prédictives très limitées, et, en second, le fait qu'ils ne fournissent pas d'interprétation physiologique des paramètres. Cependant, certains de ces modèles descriptifs peuvent proposer une certaine interprétation physiologique. Les modèles pharmacocinétiques
(PK) appartiennent à cette catégorie dans la mesure où, s'ils se présentent sous la forme de sommes d'exponentiels censés représenter des transferts entre compartiments qui n'ont aucune signification physiologique, ils permettent par contre le calcul de la clairance qui elle a une interprétation physiologique. Dans le même ordre d'idées, la paramétrisation hyperbolique du modèle pharmacodynamique (PD) du $\mathrm{E}_{\max }$ (effet maximum) provient de la théorie des récepteurs in vitro qu'il n'y a plus lieu de considérer lorsqu'on mesure une réponse sur un organisme entier. Cependant, ce modèle permet le calcul de la concentration produisant $50 \%$ de variation de l'effet maximum, qui est relié à la notion de puissance de la molécule, définie comme le rapport entre les constantes de vitesse d'association et de dissociation entre les molécules et le recepteur. ${ }^{[14,15]}$ C'est vrai pour les fonctions hyperboliques utilisées dans les modèles d'action directs comme dans les modèles indirects. ${ }^{[11,16]}$ En ce sens, modèles compartimentaux et du $\mathrm{E}_{\max }$ se situent entre modèle descriptif et mécanistique. Cependant leurs performances limitées en termes d'extrapolation plaident en faveur du développement de réels modèles mécanistiques pour l'utilisation en simulation.

\subsection{Modèles mécanistiques}

Les modèles mécanistiques se réfèrent à des jeux d'équations, chacune décrivant un processus physiologique, soit au niveau d'une cellule soit d'un organe. On parle alors de modèles physiologiques ou de modèles phénoménologiques en ce sens qu'ils permettent de construire un modèle qui n'a besoin que d'un nombre restreint de paramètres pour décrire un phénomène biologique. Les exemples classiques sont les modèles PK physiologiques (PBPK) qui modélisent les flux et échanges entre différents organes. ${ }^{[12]}$ De manière similaire, des modèles ont été développés pour de nombreux processus pharmacodynamiques, en particulier ceux impliquant des neurotransmetteurs, hormones ou toute autre molécule endogène. ${ }^{[11,17-20]}$ La plupart du temps, ces modèles nécessitent la mise en œuvre d'équations différentielles ordinaires ou stochastiques, chaque jeu d'équations décrivant des vitesses de transfert entre organes ou cellules, en accord avec des cinétiques linéaires ou non linéaires. Il faut noter que plus les équations sont proches du modèle physiologique sous-jacent, par exemple au niveau cellulaire, plus la quantité de données nécessaires à l'identification du modèle sera importante. Par exemple, les modèles PBPK nécessitent des données de concentration provenant de différents organes, ce qui implique le sacrifice du sujet d'expérience - ce qui n'est possible que sur de petits mammifères. Un des niveaux d'intégration les plus élevés atteints par ces modèles nous est fourni par le projet Physiome : « $[\ldots] a$ multicentric integrated program to design, develop, implement, 
test and document, archive and disseminate quantitative information, and integrative models of the functional behaviour of molecules, organelles, cells, tissues, organs, and intact organisms from bacteria to man $» .{ }^{[8]} \mathrm{A}$ ce niveau de complexité, il est illusoire de prétendre identifier le modèle sur des échantillons de patients dans la mesure où les données seraient trop complexes et trop chers pour être collectés lors d'un essai clinique. Conséquemment, quand il s'agit d'utiliser ces modèles pour de la simulation, des choix doivent être faits entre complexité et simplification. Une solution consiste à coupler modèles physiologiques et non physiologiques de type non paramétrique ou à identifier une partie du modèle avec les données disponibles et fixer la partie mécanistique non identifiable en utilisant une information a priori provenant de la littérature ou d'une autre expérience. ${ }^{[6,21]}$

\subsubsection{Convergence entre les deux approches}

Actuellement on assiste à une convergence de ces deux approches qui conduit à complexifier les modèles PK-PD en y intégrant de la connaissance physiopathologique, ou à simplifier une partie d'un modèle mécaniciste afin de pouvoir l'utiliser en pratique dans une simulation. Dans cette convergence, les modèles mathématiques utilisés pour représenter les données dans une démarche d'ajustement sont construits en fonction des mécanismes physiopathologiques et non plus simplement choisis de manière arbitraire, en fonction de leur simple aptitude à représenter plus ou moins bien les observations.

\subsection{Simulation des mécanismes physiopathologiques}

La somme des connaissances fondamentales sur les mécanismes physiologiques et physiopathologiques impliquées dans une maladie augmente sans cesse ainsi que les connaissances des mécanismes d'action des différents traitements. Par la nature de la démarche expérimentale, ces connaissances concernent principalement des éléments isolés des systèmes concernés : génome, cellule, tissu, organe. L'innovation et la recherche thérapeutique nécessitent d'intégrer ces connaissances dans un schéma global, le modèle thérapeutique. Actuellement, cette intégration s'effectue le plus souvent sous forme discursive. L'absence de formalisme de l'approche discursive ne permet pas la prise en compte simultanée de plusieurs dimensions. Les raisonnements discursifs sont le plus souvent mono- ou paucifactoriels et surtout non quantitatifs. Ils ne permettent pas de prendre en compte toute la complexité des conséquences d'un traitement. Les exemples où cette approche a été prise à défaut sont nombreux comme les antiarythmiques à la phase aiguë de l'infarctus, les inhibiteurs de la phosphodiestérase, etc. Le plus souvent l'intégration est parcellaire, surtout focalisée sur les bénéfices des traitements et négligeant, en partie, le métabolisme et les effets délétères. Les limites des méthodes utilisées pour la construction des modèles thérapeutiques expliquent que l'on soit conduit à entreprendre des essais thérapeutiques longs et coûteux, sur la base d'hypothèses fragiles et ne prenant pas en compte la totalité des connaissances disponibles.

\subsection{Simulation d'essais cliniques et patients " virtuels"}

Afin d'effectuer une simulation d'essais cliniques, plusieurs types de modèles sont nécessaires, afin de décrire le traitement et la maladie ; parmi ceux-ci les modèles PK et PD de population ainsi que de réponse clinique, la réponse placebo, les profils d'observance, les perdus de vue et l'analyse statistique en conjonction avec le plan d'expérience de l'essai clinique. ${ }^{[22]}$ La figure 1 exemplifie certaines de ces interactions complexes qu'on peut trouver entre les modèles traitement-maladie et les modèles d'écarts au protocole. Ces modèles doivent être ensuite appliqués à ce qu'il est convenu d'appeler des patients « virtuels ». Il s'agit là d'un abus de langage, puisque ces patients ne sont définis que par quelques covariables (âge, sexe, poids, génotype ou phénotype, biomarqueur) qui n'expliquent pas toute la complexité des interactions entre la biologie d'un organisme, la maladie et le traitement. Ces covariables peuvent être soit générées par un modèle ou ré-échantillonnées à partir d'une base de données existante. Par exemple, la répartition des sexes peut facilement être simulée à partir d'une distribution binomiale et l'âge d'une distribution log-normale tronquée. Cependant, quand des covariables physiopathologiques sont introduites, les corrélations entre covariables ne peuvent être ignorées. Typiquement, la taille et le poids sont corrélés avec le sexe, et la fonction rénale avec l'âge. De plus, ces covariables peuvent avoir des distributions multimodales parfois liées à des différences génétiques. Le problème se complexifie lorsque les covariables varient au cours du temps, (p. ex. un biomarqueur de l'ostéoporose), ce qui nécessite un modèle longitudinal pour décrire ces changements en plus des corrélations. ${ }^{[23]}$ Plus grand est le nombre de covariables introduites, plus réaliste le « patient virtuel » est censé être, mais aussi plus grand est le risque de simuler des patients hautement improbables du fait de l'ignorance de toutes les complexités biologiques. Une alternative à la simulation de "patients virtuels » est l'utilisation de base de données épidémiologiques d'où on ré-échantillonne des patients réels. Un exemple nous est donné dans une simulation d'essai clinique pour l'angine de poitrine où, plutôt que de simuler des profils longitudinaux de fréquence cardiaque, prédictifs du risque d'angine de poitrine, ces fréquences cardiaques étaient ré-échantillonnées de la base de données épidémiologique OCTAVE II (Optimisation du Contrôle de la Tension Artérielle, Valeur de l'Enregistrement sur 


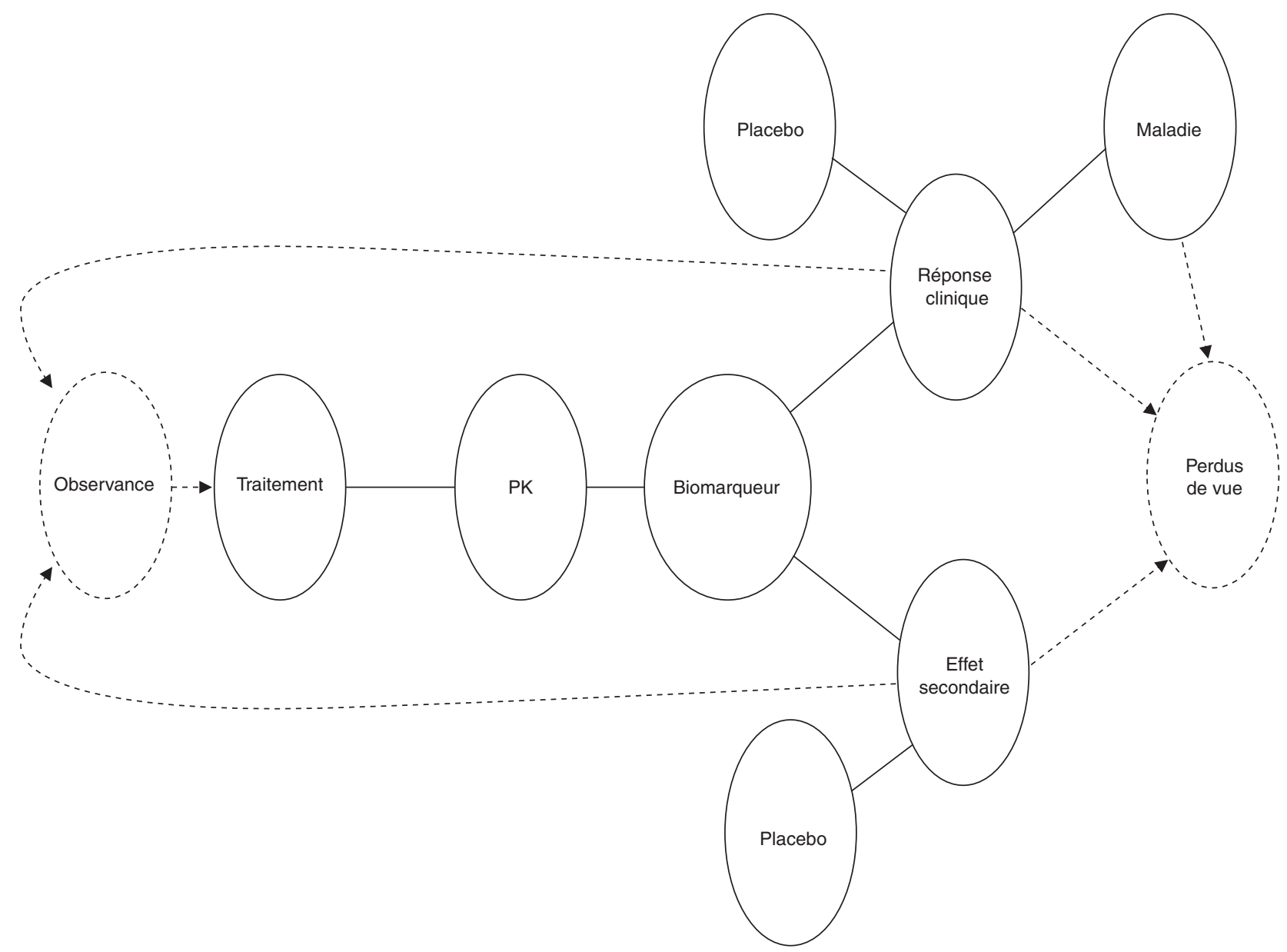

Fig. 1. Représentation schématique des interactions entre traitement, maladie et écarts au protocole figurés ici par la non-observance et la sortie prématurée d'étude. Les modèles de causalité entre traitement et maladie sont en traits pleins, tandis que les modèles d'écarts au protocole en traits pointillés. II est à noter que l'observance peut influencer la pharmacocinétique (PK) ou la pharmacodynamie (PD) qui, par un mécanisme de « feedback », peuvent à leur tour modifier l'observance.

24 h). ${ }^{[6]}$ Cette technique conduit à la création de patients dits « hybrides » qui sont en partie caractérisés par des données rééchantillonnées et en partie par des données simulées.

\section{Périmètre de l'utilisation de la modélisation et simulation (M\&S)}

\subsection{Objectifs et bénéfices}

La modélisation - simulation (M\&S) est un outil générique qui peut être utilisé à différents niveaux du développement des médicaments : premièrement en préclinique (aussi bien pour la relation structure activité que pour les études de toxicité), comme preuve de concept en utilisant une modélisation mécanistique pour vérifier les hypothèses thérapeutiques; deuxièmement dans l'extrapolation de l'animal à l'homme en utilisant les modèles allométriques et dans la prédétermination des doses en phase I ; et finalement en phases II et III pour l'optimisation des protocoles et l'analyse de décision (voir paragraphes 3.3 et 3.4).

Le principal objectif de la M\&S est d'apporter des bases quantitatives à l'analyse décisionnelle. Les décisions prises sur la base de modélisation et simulation vont de l'amélioration du design des essais cliniques jusqu' au rejet des études peu utiles ou celles qui pourraient potentiellement échouer et de la réalisation d'essais plus rapides et/ou plus économiques jusqu'à l'arrêt du développement.

Le bénéfice secondaire de la $M \& S$ est de rassembler des personnes des différents domaines du développement des médicaments (pharmacologues, cliniciens, pharmacométriciens, statisticiens, développeurs de médicaments, etc.), et de faciliter et promouvoir leurs collaborations en les incitant à partager leurs connaissances à travers des modèles, la formalisation mathé- 
matique des hypothèses et par une meilleure connaissance de toutes les dimensions du développement. Dans ce sens, la M\&S et la simulation des essais cliniques sont des outils de communication de grande valeur pour les firmes pharmaceutiques.

\subsection{Preuve de concept}

L'approche «in silico » permet de construire des modèles thérapeutiques complexes intégrant la quasi-totalité des connaissances disponibles sur les mécanismes de la maladie et sur ceux des traitements. Grâce à ces modèles numériques et par simulations d'essais thérapeutiques, on peut tester la vraisemblance des hypothèses et réaliser ainsi une preuve de concept.

Alors que les pistes pour l'innovation thérapeutique deviennent plus nombreuses avec les progrès de la biologie moléculaire et de la génétique, les arrivées sur le marché de nouveaux médicaments se ralentissent. ${ }^{[24]}$ D'une part la seule épreuve de réfutation du modèle thérapeutique sur lequel repose l'innovation est l'essai thérapeutique, et la vérification expérimentale du modèle thérapeutique, par l'essai thérapeutique, est un processus lourd, long, coûteux et très aléatoire, d'autre part les pièges d'un métabolisme particulier ou d'effets indésirables rédhibitoires ne peuvent être que faiblement anticipés par les études sur modèle animaux. L'expérimentation « in silico » devrait permettre une prévalidation du modèle thérapeutique permettant ainsi une preuve du concept avant la prise de décision de lancer le processus de développement clinique. L'intégration des mécanismes d'action des traitements et des mécanismes physiopathologiques des maladies au sein de modèles numériques permet d'obtenir des modèles thérapeutiques plus fiables que ceux obtenus de manière discursive. Les modèles mathématiques devraient permettre par simulations informatiques d'étudier les bénéfices cliniques de la même façon que l'essai thérapeutique.

\subsection{Optimisation des études cliniques}

L'optimisation des études cliniques consiste à rechercher des plans d'étude qui maximisent l'efficacité et minimisent la toxicité (meilleur rapport efficacité/toxicité) pour le coût le plus faible. Pour cela, des simulations d'essais cliniques sont réalisées en faisant varier différentes caractéristiques de l'étude : classiquement le nombre de sujets par bras, la dose ou le schéma posologique, ou, de façon moins classique, le plan d'expérience (groupes parallèles ou essai en cross over), le nombre de visites par patient, le choix du critère de jugement, le choix du comparateur (placebo ou produit actif), la durée de l'étude, l'impact des critères d'inclusion ou des points de logistique ou pharmacoéconomie. ${ }^{[25]}$

\subsection{Analyse décisionnelle et développement du médicament}

L'objectif de l'analyse décisionnelle est d'aider les décisionnaires à appréhender de manière systématique des problèmes complexes et d'améliorer la qualité des décisions résultantes. Une décision typique est celle d'arrêter ou de continuer le développement quand une certaine quantité d'informations a été amassée. Les méthodes d'aide à la décision quantitative peuvent être utiles quand les décisions sont difficiles en raison d'une complexité sous-jacente, quand il existe un certain degré d'incertitude sur les conséquences des décisions, quand les décisions ont de multiples objectifs ou quand différentes perspectives peuvent conduirent à différentes décisions. Un processus d'aide à la décision quantitative peut être décomposé en une succession d'étapes : (i) identifier la décision ; (ii) identifier les alternatives ; (iii) identifier les conséquences ; (iv) modéliser les décisions ; (v) évaluer les alternatives ; et (vi) synthétiser les étapes précédentes et recommencer si nécessaire.

Typiquement la M\&S apporte un outil pour construire un modèle exposition-réponse intégré pour l'étape (iv). ${ }^{[25]}$ Les arbres de décision apportent une représentation claire de la structure des décisions : la séquence décisionnelle, les alternatives, l'incertitude, les conséquences (voir figure 2).

\subsection{Tester les sous-groupes et compléter le dossier d'AMM}

Une autre application possible est de compléter le dossier d'enregistrement en documentant un aspect des effets d'un traitement (p. ex., répondre à des questions concernant des sousgroupes, déterminer l'impact de la non-observance, etc.) ou répondre à des questions à partir des données déjà accumulées sans avoir à entreprendre de nouvelles études. Cette approche ne semble vraiment envisageable que dans quelques situations où la réponse aux questions se posant est inaccessible par des études ou des interrogations accessoires ne concernant pas directement la démonstration de l'efficacité.

Une autre utilisation de la simulation des essais cliniques est de compléter le dossier d'un nouveau médicament et de documenter des aspects non étudiés. Un exemple typique combinant ces deux utilisations de la simulation des essais cliniques est donné par la prédiction de l'effet de docétaxel chez un sousgroupe de patients particuliers. ${ }^{[5]} \mathrm{Il}$ a été montré que les patients porteurs d'un cancer du poumon non à petites cellules avec un taux élevé d' $\alpha 1$ glycoprotéine acide avaient un temps de progression et de décès réduit. Pour évaluer dans quelle mesure ces patients pourraient bénéficier d'une intensification de dose, des simulations ont été réalisées et ont montré la faible puissance 

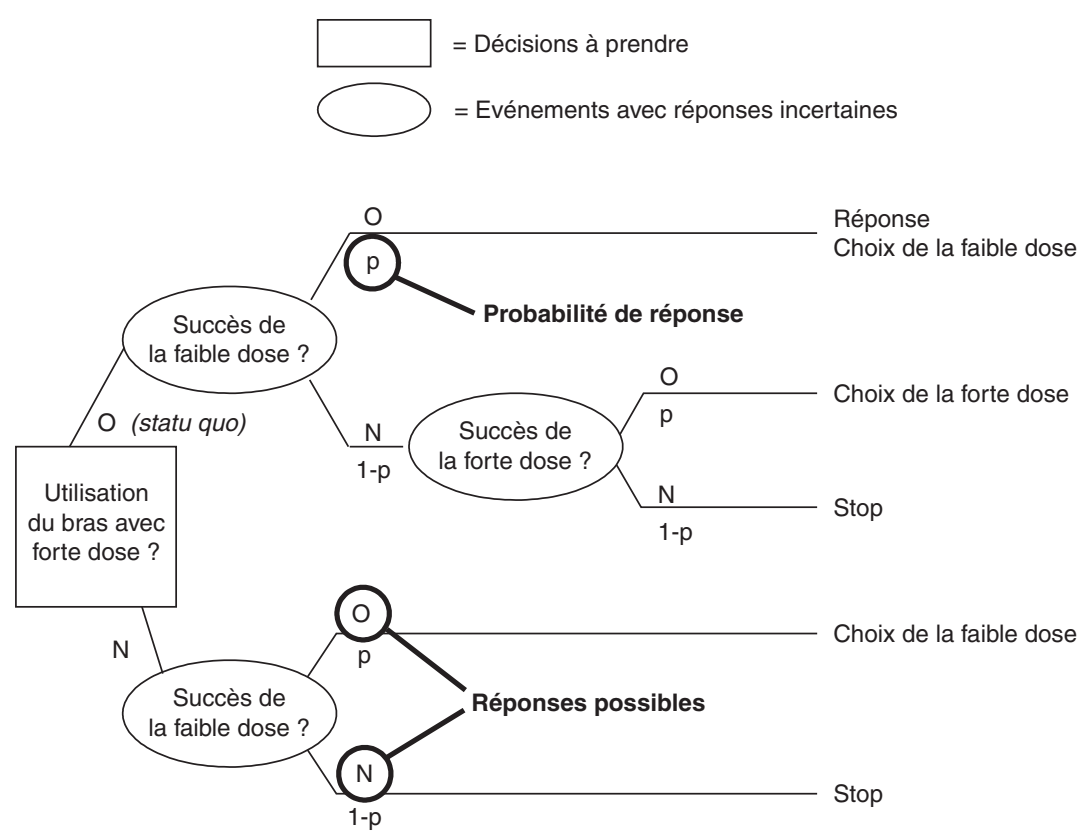

Fig. 2. Arbre de décision montrant la structure d'une décision. $\mathbf{O}=$ oui ; $\mathbf{N}=$ non.

d'une telle étude si elle était entreprise. Cela a conduit à la décision de ne pas réaliser d'étude sur ce sous-groupe de population. ${ }^{[5]}$ Cependant, une fois de plus, un large consensus s'est dégagé de la table ronde sur le fait qu'en aucune manière un résultat de simulation ne pouvait remplacer un essai clinique réel.

\subsection{Modèle d'écart au protocole}

Quand un essai est planifié, il est généralement supposé qu'il sera exécuté conformément à un protocole spécifique qui décrit tous les aspects expérimentaux du début à la fin de l'étude. Par exemple, les modalités de suivi des patients doivent être spécifiées avec précision dans tous les protocoles et définir : les caractéristiques des patients (critères d'inclusion et exclusion), le nombre de sujets, les traitements et les méthodes d'allocation, le régime thérapeutique (dose et fréquence d'administration), les mesures à effectuer (type, date et temps), la durée de l'étude.

L'adhérence au protocole doit permettre l'estimation des conséquences du traitement (efficacité et innocuité) avec suffisamment de puissance statistique, ou tout du moins avec celle qui est attendue. En réalité, cependant, les écarts au protocole peuvent empêcher l'étude d'atteindre les objectifs qui lui ont été assignés. Par anticipation des écarts au protocole qui contribuent à une inflation de la variabilité résiduelle et diminuent la puissance statistique, les concepteurs d'essais ont tendance à surdimensionner les études de façon arbitraire. Il peut être mis en évidence que dans certain cas l'augmentation du nombre de sujets est insuffisante pour compenser cette diminution de puissance et que dans d'autre cas ce surdimensionnement peut conduire à des essais inutilement trop grands avec des conséquences financières notables sur le programme de développement. Il est difficile d'estimer quantitativement les conséquences d'un seul écart au protocole sur la puissance statistique et, a fortiori, il est presque impossible de le faire pour la combinaison de plusieurs écarts. La seule façon d'estimer les écarts au protocole est d'utiliser les techniques de modélisation et de simulation et plus spécifiquement les modèles stochastiques longitudinaux, qui sont le seul moyen de décrire les comportements individuels et d'étudier l'impact de ces déviations au protocole en utilisant des scénarios de type «que se passe-t-il si... »:[26]

- que se passe-t-il si les critères d'inclusion/exclusion ne sont pas suivis?

- que se passe-t-il s'il y a moins de patients inclus qu'attendu ?

- que se passe-t-il si les patients ou les investigateurs ne suivent pas les dates de visites?

- que se passe-t-il s'il y a des données manquantes ?

- que se passe-t-il si les patients prennent des doses insuffisantes ou des doses supplémentaires, mais à l'horaire prévu?

- que se passe-t-il si des patients arrêtent de prendre leur traitement?

- $\quad$ que se passe-t-il si des patients sortent de l'étude ? 


\section{Méthodologie}

La méthodologie de la simulation des essais cliniques a maintenant été largement abordée dans plusieurs articles, un livre et un document publié sur le web. ${ }^{[7,22]}$ La première étape de la réalisation d'une simulation d'essai clinique est de définir les objectifs et le niveau de complexité du modèle que l'équipe de simulation veut atteindre. La deuxième étape est de faire la liste des hypothèses, de construire le modèle et de le qualifier. ${ }^{[27]} \mathrm{La}$ construction du modèle implique l'identification de la structure des différents modèles (PK, PD, des conséquences cliniques et toxiques) et aussi des éléments de variabilité inter- et intra-patients et d'incertitude des modèles. La variabilité inexpliquée est estimée conditionnellement aux données existantes en utilisant des modèles non linéaires à effets mixtes. De la même manière, la précision avec laquelle sont estimés les paramètres du modèle est prise en compte. Cette précision dépend en premier de la quantité d'informations disponibles et de la qualité du modèle (absence d'erreur de spécification du modèle). Par exemple, dans le cas d'un modèle parfait, la précision tend vers l'infini et l'erreur vers zéro quand la quantité d'informations tend vers l'infini. La méthodologie des modèles non linéaires à effets mixtes procure par défaut des estimations de l'incertitude. Ignorer cette incertitude conduirait à une surestimation systématique du taux de succès. Lorsque aucune donnée n'est disponible, ou alors qu'une partie du modèle ou la variabilité et l'incertitude n'ont pas été estimées, ils peuvent être fixés à partir d'informations a priori, venant d'études précédentes ou de la littérature scientifique, ou imputés après avoir fait une analyse de sensibilité de tous les paramètres pertinents du modèle.

L'étape suivante consiste en la construction et la réalisation des simulations, qui se décompose en : (i) écrire un protocole de simulation qui englobe le protocole d'analyse des études simulées ; (ii) simuler des patients « virtuels » ou hybrides (voir paragraphe 2.5) ; (iii) créer de multiples réplications d'études simulées au travers de l'incertitude ; (iv) représenter les résultats en termes de distributions et tables; et (v) réaliser les analyses de sensibilité.

La validation d'une simulation se décompose en validation interne (est-ce que ma simulation reproduit les données utilisées pour construire le modèle ?) et en validation externe par confrontation à de nouvelles données observées qui n'ont pas été utilisées pour la construction du modèle initial.

La plupart du temps, cette dernière validation ne peut seulement être effectuée qu'a posteriori puisque par définition la simulation des essais cliniques est utilisée pour prédire les résultats d'un essai non encore réalisé.

\section{Bilan sur les pratiques courantes}

\section{1 Sondage Giens 2003}

Un rapide sondage a été réalisé auprès des participants des Journées de Giens 2003 (46 réponses/153 participants). Il en ressort que l'approche modélisation et simulation des essais cliniques existe déjà, de façon marginale, à l'université et aussi dans l'industrie, avec comme objectif l'optimisation du développement ; le souhait majoritaire exprimé dans ce sondage, mais qu'il faut nuancer du fait de sa faible représentativité, est de voir cette approche se développer dans le futur.

\subsection{Exemple}

L'ivabradine est un nouvel agent qui diminue la fréquence cardiaque avec comme indication potentielle l'angine de poitrine stable. Afin d'investiguer son meilleur compromis entre efficacité et innocuité, sa posologie et le nombre de patients à inclure dans une phase III, une simulation d'essais cliniques a été exécutée, utilisant un modèle thérapeutique complet. ${ }^{[6,28]}$ Cet exemple illustre plusieurs aspects de la simulation d'essais cliniques : création de «patients hybrides », utilisation d'une information a priori provenant de la littérature pour construire le modèle clinique. En bref, le critère binaire, crise d'angine, fut simulé à l'aide d'un modèle physiologique dans lequel la réserve coronaire se calcule à partir de la fréquence cardiaque. L'innocuité fut définie comme l'absence de bradycardie excessive. A l'aide de données de phase I, un modèle PK-PD permettant de prédire les baisses de fréquence cardiaque en fonction des concentrations a été développé et, après ré-échantillonnage de profils de fréquence cardiaque d'une base de données épidémiologiques, 100 essais cliniques ont été simulés, chacun incluant 100 patients dans le groupe contrôle et 100 recevant une dose orale $(2,5 ; 5 ; 10 ; 20$ et $40 \mathrm{mg}$ [dose quotidienne] ou 2 fois/jour) d'ivabradine. Seulement $25 \%$ des essais simulés montrèrent une différence significative en faveur de l'ivabradine avec la dose $10 \mathrm{mg} 1$ fois/jour, $48 \%$ et $55 \%$ avec les doses 10 $\mathrm{mg} 2$ fois/jour et $20 \mathrm{mg} 1$ fois/jour, respectivement, et plus de $80 \%$ de succès avec la dose $40 \mathrm{mg} 1$ fois/jour. Quatre pour cent des patients montraient au moins un effet indésirable dans le groupe contrôle, et de 5 à $13 \%$ dans les groupes traités de la plus faible à la plus forte dose. Sur la base de ces résultats de simulation, les auteurs ont calculé que le nombre de sujets à inclure dans une future étude afin d'obtenir une baisse de $15 \%$ des crises d'angine de poitrine, si on suppose un risque de base de $68 \%$, serait de 239 sujets par groupe pour la dose de $10 \mathrm{mg} 2$ fois/jour ou de 196 pour celle de $20 \mathrm{mg} 1$ fois/jour. Pour autant, ces résultats ayant été obtenus à partir de modèles soit établis chez le 
volontaire sain en phase I soit publiés dans une revue scientifique n'ont qu' une valeur indicative et demandent encore à être validés par des données réelles.

\section{Qu'est-ce qui peut freiner, accélérer le développement de la M\&S}

La table ronde a cherché à identifier les points sur lesquels les efforts devront porter à l'avenir afin de permettre l'intégration de l'approche de modélisation - simulation dans le développement standard des traitements. Le premier facteur est la nécessité que les acteurs du domaine, industriels et académiques, s'approprient la méthodologie. Pour cela il est impératif que des exemples, apportant la preuve de concept, soient publiés, malgré la confidentialité des données. Ces publications sont indispensables pour faire connaître l'approche, concrétiser les déclarations de principes, et analyser ses intérêts et ses limites. La généralisation de la $\mathrm{M} \& \mathrm{~S}$ des essais cliniques implique la mise à disposition de bases de connaissances et de données spécifiques avec un accès facile à des bibliothèques de modélisation mathématique de systèmes biologiques, à des bases de données de résultats des études expérimentales et des bases de données de patients nécessaires à la réalisation des simulations. Il conviendra aussi d'obtenir des ressources pour initier des équipes pluridisciplinaires dédiées, de former les acteurs et les équipes à la multidisciplinarité, d'organiser les nombreuses compétences nécessaires par exemple en les « dé-compartimentant». La réalisation pratique de ces $\mathrm{M} \& \mathrm{~S}$ pourrait être grandement facilitée par la disponibilité d'un logiciel bivalent, modélisation et simulation, permettant de créer de façon générique les modèles, de réaliser dans la foulée les simulations puis de revenir le cas échéant à l'étape de modélisation.

Au final, l'édification de recommandations de bonnes pratiques de modélisation - simulation semble incontournable. D'ores et déjà un document de travail, réalisé sous l'égide du CDDS (Center for Drug Development Science), existe et un travail de ce type a été initié à l'INSERM (Institut national de la santé et de la recherche médicale) et tous les deux nécessitent maintenant d'être complétés. ${ }^{[22]}$

\section{Conclusion}

Dans de nombreux domaines comme la cardiologie, la cancérologie, l'ostéoporose, la polyarthrite rhumatoïde, les essais cliniques nécessitent de grands nombres de patients et des durées de suivi importantes. Le risque d'essais négatifs doit être limité au maximum, ce qui implique que les essais soient basés sur des hypothèses les plus solides possibles. L'utilisation des modèles mécanistiques et de la simulation des essais cliniques ouvre la porte à un nouveau paradigme. Désormais la réfutation des hypothèses ne se trouve plus cantonnée aux expériences «in vitro » ou «in vivo » mais peuvent aussi faire appel à des modèles numériques et informatiques, pouvant être qualifiés d'expériences «in silico ».

\section{Participants}

J.-P. Boissel (EA643 SPC Université Lyon 1), M. Cucherat (EA643 SPC Université Lyon 1), S. Durrleman (Sanofi-Synthelabo, Chilly Mazarin), P. Girard (INSERM et EA3738 CTO Université Lyon 1), D. Guez (Laboratoires Servier, Courbevoie), R. Koen (Pharmacia SAS, St Quentin en Yvelines), C. Laveille (Laboratoires Servier, Courbevoie), H. Mathiex-Fortunet (Beaufour IPSEN Pharma, Paris), J. Micallef (CHU La Timone CPCET, Marseille), N. Missoum (Merck Sharp \& Dohme, Paris), G. Paintaud (Hôpital Bretonneau Pharmacologie, Tours), M.-C. Perault (CIC CHU, Tours), M. Tansey (Pharmacia, Jersey City, USA), J.-L. Thomas (Merck Santé, Lyon), J.-M. Treluyer (Hôpital St Vincent de Paul Pharmacologie Clinique, Paris), Ph. Variol (Pierre Fabre Médicament, Castres), T. Waegemans (L.F.B., Paris).

\section{Références}

1. Brooks MM, Hallstrom A, Peckova M. A simulation study used to design the sequential monitoring plan for a clinical trial. Stat Med 1995; 14: 2227-37

2. Hale MD, Gillespie WR, Gupta SK, et al. Clinical trial simulation as a tool for increased drug development efficiency. Appl Clin Trials 1996; 5: 35-40

3. Holford NH, Kimko HC, Monteleone JP, et al. Simulation of clinical trials. Annu Rev Pharmacol Toxicol 2000; 40: 209-34

4. Kimko HC, Reele SS, Holford NH, et al. Prediction of the outcome of a phase 3 clinical trial of an antischizophrenic agent (quetiapine fumarate) by simulation with a population pharmacokinetic and pharmacodynamic model. Clin Pharmacol Ther 2000; 68: 568-77

5. Veyrat-Follet C, Bruno R, Olivares R, et al. Clinical trial simulation of docetaxel in patients with cancer as a tool for dosage optimization. Clin Pharmacol Ther 2000; 68: 677-87

6. Chabaud S, Girard P, Nony P, et al. Clinical trial simulation using therapeutic effect modeling: application to ivabradine efficacy in patients with angina pectoris. J Pharmacokinet Pharmacodyn 2002; 29: 339-63

7. Ko HC, Dufull S. Simulation for designing clinical trials. New-York, USA: Marcel Dekker, 2002

8. Bassingthwaighte JB. Strategies for the physiome project. Ann Biomed Eng 2000; 28: $1043-58$

9. Sheiner LB, Steimer JL. Pharmacokinetic/pharmacodynamic modeling in drug development. Annu Rev Pharmacol Toxicol 2000; 40: 67-95

10. What impact does the use of flight simulators have on commercial aviation? [online]. Available from URL: http://www.airlinesafety.com/faq/faq1.htm [Accessed 2004 Jul 21]

11. Jusko WJ, Ko HC. Physiologic indirect response models characterize diverse types of pharmacodynamic effects. Clin Pharmacol Ther 1994; 56: 406-19

12. Charnick SB, Kawai R, Nedelman JR, et al. Perspectives in pharmacokinetics. Physiologically based pharmacokinetic modeling as a tool for drug development. J Pharmacokinet Biopharm 1995; 23: 217-29

13. Jupp DLB. Approximation to data by splines with free knots. SIAM J Numer Anal 1978; 15: 328-43

14. Wellstein A, Palm D. Correlation between pharmacological response, kinetics of plasma concentration and in vitro receptor affinities exemplified with betaadrenoceptor blocking dr drugs. Methods Find Exp Clin Pharmacol 1984; 6: 641-4

15. Wellstein A, Palm D, Pitschner HF, et al. Receptor binding of propranolol is the missing link between plasma concentration kinetics and the effect-time course in man. Eur J Clin Pharmacol 1985; 29: 131-47 
16. Jusko WJ, Ko HC, Ebling WF. Convergence of direct and indirect pharmacodynamic response models. J Pharmacokinet Biopharm 1995; 23: 5-8

17. Kushler R, Brown B. A model for the identification of hormone pulses. Stat Med 1991; 10: 329-40

18. Camproux AC, Thalabard JC, Thomas G. Stochastic modeling of the hypothalamic pulse generator activity. Am J Physiol 1994; 267: E795-800

19. Hoffman A, Goldberg A. The relationship between receptor-effector unit heterogeneity and the shape of the concentration-effect profile: pharmacodynamic implications. J Pharmacokinet Biopharm 1994; 22: 449-68

20. Jusko WJ. Receptor-mediated pharmacodynamics of corticosteroids. Prog Clin Biol Res 1994; 387: 261-70

21. Troconiz IF, Sheiner LB, Verotta D. Semiparametric models for antagonistic drug interactions. J Appl Physiol 1994; 76: 2224-33

22. Holford NH, Hale MD, Ko HC, et al. Simulation in drug development: good practices. Draft Publication of the Center for Drug Development Science (CDDS). Draft version 1.0, 23 Jul, 1999 [online]. Available from URL: http://cdds. georgetown.edu/research/sddgp723.html [Accessed 2004 Jul 19]

23. Greenland S. Interpreting time-related trends in effect estimates. J Chronic Dis 1987; 40 Suppl. 2: 17S-24S
24. US Food and Drug Administration. US Department of Health and Human Services. Innovation or stagnation: challenge and opportunity on the critical path to new medical products [online]. Available from URL: http://www.fda.gov/oc/initiatives/criticalpath/whitepaper.html [Accessed 2004 Jul 29]

25. Wada DR, Poland B. Combining drug-disease and economic modelling to inform drug development decisions. Drug Discov Today 2001; 6: 1165-70

26. Kastrissios H, Girard P. Protocol deviations and execution models. In: Ko HC, Duffull SB, editors. Simulation for designing clinical trials. New York, USA: Marcel Dekker, 2002

27. Yano Y, Beal SL, Sheiner LB. Evaluating pharmacokinetic/pharmacodynamic models using the posterior predictive check. J Pharmacokinet Pharmacodyn 2001; 28: 171-92

28. Duffull SB, Chabaud S, Nony P, et al. A pharmacokinetic simulation model for ivabradine in healthy volunteers. Eur J Pharm Sci 2000; 10: 285-94

Correspondance et offprints : Pascal Girard, INSERM et EA 3738 Ciblage Thérapeutique en Oncologie, Faculté de Médecine Lyon-Sud, BP12, 69921 Oullins Cedex, France.

E-mail : Pascal.Girard@adm.univ-lyon1.fr 